\title{
Adverse Drug Reaction Profile and Medication Chart Review in Orthopaedic Patients Receiving Diclofenac Sodium at a Tertiary Care Urban Hospital
}

\author{
K. Sravani ${ }^{1}$, Dr. Shaik Kareemulla ${ }^{2 *}$, S. Mohammed Ishak ${ }^{1}$, N. Ashwini ${ }^{1}$
}

${ }^{1}$ Pharm D Intern, Department of Pharmacy Practice, P. Rami Reddy Memorial College of Pharmacy, Kadapa, Andhra Pradesh, India

${ }^{2}$ Assistant Professor, Department of Pharmacy Practice, P. Rami Reddy Memorial College of Pharmacy, Kadapa, Andhra Pradesh, India

*Corresponding author: Dr. Shaik Kareemulla

Abstract

Background: Diclofenac is the most commonly prescribed drug in clinical settings for the management of pain and inflammation. Gastrointestinal toxicity is a major clinical limitation of Diclofenac. A number of studies describe NASIDs as the leading causes of adverse drug reactions (ADRs). Objectives: The main aim of this study was to evaluate the prescribing pattern of Diclofenac and to monitor the ADRs in orthopaedic IP patients with various comorbidities. Materials \& Methods: A prospective observational study was planned and conducted in the Department of Orthopaedics IPD of a tertiary care teaching hospital, RIMS. Patient data relevant to the study was obtained using a standard data collection form designed. A predesigned proforma of CDSCO (central Drug Standards Control Organisation) was used to record ADR. The causality was analyzed by using WHO causality assessment scale and the severity was analyzed by using the Hartwing and Siegel scale. Results: NSAID (Diclofenac) was commonly prescribed for osteoarthritis (10\%), rheumatoid arthritis (11.67\%), Fractures (45\%), lower backache (10\%), Spondylosis $(8.33 \%)$, and osteomyelitis $(15 \%)$. Nearly $63.64 \%(\mathrm{n}=7)$ of the ADRs were reported by men and $36.36 \%$ $(\mathrm{n}=3)$ were reported by females. The reactions which were observed were nausea, vomiting, gastritis, abdominal discomfort, diarrhoea and headache. The most commonly reported ADR was gastritis and the system which was involved was the gastro-intestinal system with diclofenac. As per WHO causality assessment scale, 11.67\% ADRs were assessed as "possible" ADRs and 5\% as "probable" ADRs. The severity assessment scale revealed that $100 \%$ were mild and that moderate and severe ADRs were not identified. Conclusion: Knowledge of potential adverse effects and standard prescription guide lines will play pivotal role in rational prescription of NSAIDs (diclofenac). Regular prescription audits with feedback to prescribers can alter prescribing behaviour towards good prescribing practices.

Keywords: Diclofenac, Gastritis, Hartwig and siegel scale, Fractures.

Copyright @ 2020: This is an open-access article distributed under the terms of the Creative Commons Attribution license which permits unrestricted use, distribution, and reproduction in any medium for non-commercial use (NonCommercial, or CC-BY-NC) provided the original author and source are credited

\section{INTRODUCTION}

Inflammatory disorders like both arthritic and non-arthritic conditions are most commonly encountered in orthopaedic department and these conditions are generally treated with NSAIDs (mostly diclofenac) with or without additional or specific therapies like steroids, immunomodulators or disease modifying anti-rheumatic drugs [1]. Fractures are among the most common orthopaedic problems, and about 6.8 million people seek medical care attention for fracture in India and most common indication for prescribing diclofenac was fractures. The usage of NSAIDs is mainly empirical as they provide only symptomatic relief without addressing the underlying process. Nonsteroidal anti-inflammatory drugs are the most commonly prescribed group of drugs in clinical settings for the management of pain and inflammation. Diclofenac is the most widely used medication worldwide over the counter and also it is the only drug which happen to be the most widely prescribed and often misused as self-medication even for trivial complaints. Diclofenac is a Non-selective COX inhibitor. Usually NSAIDs work by interfering with cyclooxygenase [COX] pathway, which involves the conversion of arachidonic acid by the enzyme COX to prostaglandins. COX is available in two isoforms i.e. COX-1 and COX-2. The COX-1 enzyme is constitutive and control physiological functions such as stomach mucus production and kidney water excretion as well as 
platelet formation. In contrast, COX-2 is involved in producing prostaglandins for inflammatory response. Despite wide clinical use of classical NSAIDs as analgesics, anti-pyretics, and anti-inflammatory agents their gastro-intestinal toxicity is a major clinical limitation [2]. This adverse effect is associated with their ability to inhibit COX-1 in the GIT. Subsequently, the selective COX-2 inhibitors emerged as potentially gastro-friendly NSAIDs and it was conceptualized that sufficient therapeutic benefits are achieved by selective COX-2 inhibition. At first glance these COX-2 inhibitors look like solution to NSAIDs related GI complication. However, Post marketing experience unmarked various adverse cardiovascular effects. Recent evidences of adverse CVS events with the use of COX-2 selective inhibitors have created a sense of insecurity not only among prescribers but also among consumers [3]. With variety of NSAIDs that are presently available, it is difficult at times to select a particular NSAID on a rationale basis alone but on empiricism. These are mostly used for variety of indications like rheumatoid arthritis (RA), osteoarthritis (OA), low back pain (LBP) et [4].

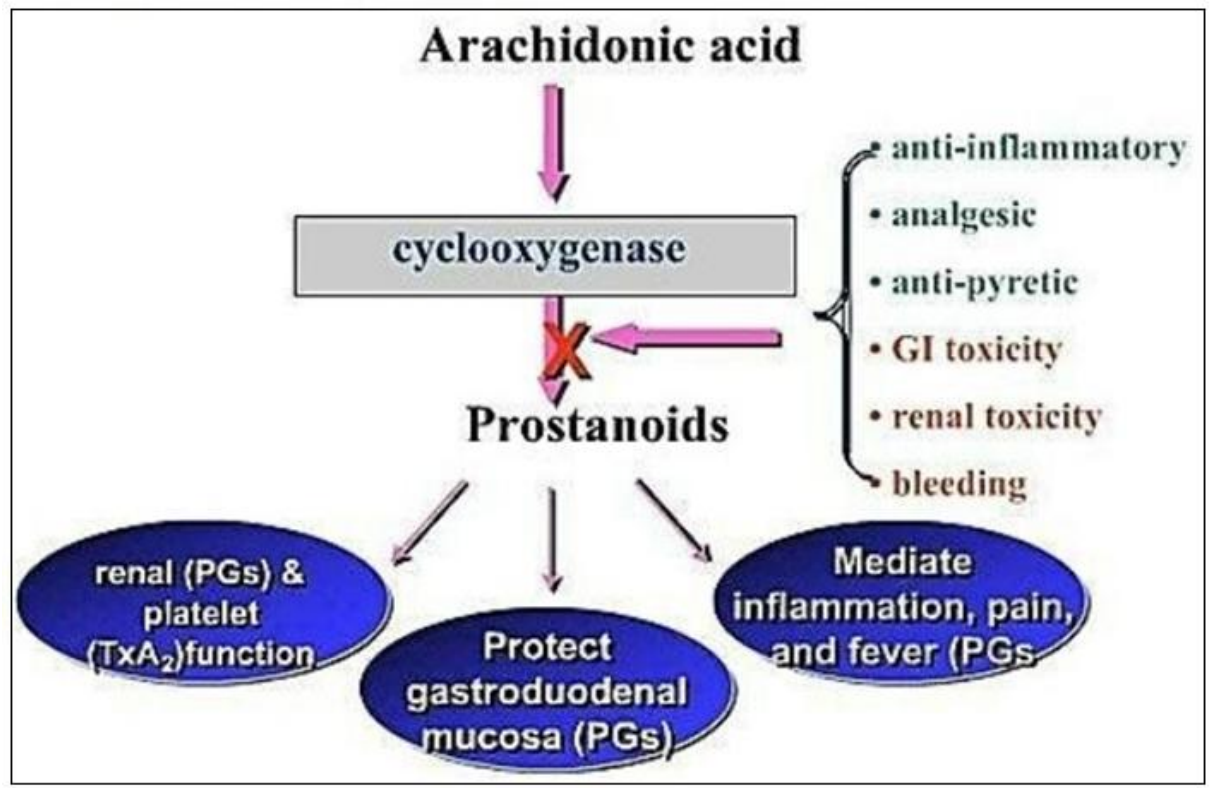

Fig-1: Shows mechanism of action of Diclofenac

An 'adverse drug reaction', as defined by the World Health Organization, is a noxious, unintended effect of a drug, which occurs at normal doses in humans for the prophylaxis, diagnosis, or the therapy of the disease or for the modification of its physiological function. ADRs are considered as the 4th to 6th leading causes of death among hospitalized patients. These are associated with significant morbidity, mortality and permanent disability and are a huge economic burden on the patients due to prolonged hospitalization. It has been estimated that the incidence of ADRs throughout the world is $5 \%$. In south Indian hospitals, ADRs accounted for $0.7 \%$ of the total admissions and $1.8 \%$ of the total deaths. An important risk factor for developing $\mathrm{ADR}$ is the previous occurrence of ADR. Re-exposure to offending drugs due to poor documentation can cause the patient to experience the same ADR again, thus emphasizing the importance of the accurate documentation of ADR at the time of the event and providing relevant information to the patient about the ADR to help prevent its further occurrence [5]. Gastrointestinal toxicity is a major clinical limitation of Diclofenac. Therefore, periodic evaluation of drug utilization patterns needs to be done to enable suitable modifications in the prescription of drugs to increase the therapeutic benefit and decrease the adverse effects. Prescribing pattern studies are conducted to monitor, evaluate and if necessary, suggest modifications in the prescribing behaviour of medical practitioners to make medical care rational and cost effective. Rational use of medicines (RUM) is an issue that has global importance, as it aims at evaluating the accessibility, availability, and correct prescribing of the drugs. In developing countries like India, where the financial resources are scarce and affordability of the patients is less, implementation of RUM becomes more important and therefore, the assessment of prescribing pattern is vital for clinical, economic, and educational purposes [4].

\section{AIM OF THE STUDY}

To evaluate the prescribing pattern of Diclofenac and to monitor the ADRs in orthopaedic IP patients with various co- morbidities.

\section{MATERIALS AND METHODS}

Keeping present scenario in mind, a prospective observational study was planned and conducted in the Department of Orthopaedics IPD of a tertiary care teaching hospital, Rajiv Gandhi Institute of 
Medical Sciences (RIMS), Kadapa, Andhra Pradesh in 2019 to analyze the prescribing pattern of NSAIDs (Diclofenac).

Following details were recorded from each prescription:

1. Patient's demographic details.

2. Details about patient's disease.

3. Concomitant illness.

4. Treatment details.

A predesigned proforma of central drugs standards control organisation was used to record ADR. The outcome parameters were types of ADR, symptomatic treatment required for ADR, requirement of dechallenge, and whether rechallenge was done, if ethically allowed. Causality assessment for the adverse drug reactions was done as per the WHO causality assessment Uppsala monitoring centre scale.

ADRs were graded as mild, moderate and severe as follows. Mild: transient or mild discomfort; no limitation in activity; no medical intervention/therapy required. Moderate: limitation in activity-some assistance may be needed; medical intervention/therapy required. Severe: marked limitation in activity; some assistance usually required; medical intervention/therapy required; hospitalisation possible. Severe: life-threatening- extreme limitation in activity, significant assistance required; significant medical intervention/ therapy required; hospitalisation is necessary.

\section{Inclusion Criteria}

1. Patients age $\geq 21$ years

2. Orthopedic patients with any co morbid conditions.

3. In patients were included in the study.

\section{Exclusion Criteria}

1. Patients age more than 70 years were excluded from the study

2. Pregnant women were excluded from the study.

3. Patient who are not willing to participate in the study.

4. Out patients were excluded from the study.

\section{RESULTS}

This Prospective observational study was conducted in orthopaedics department for a period of 100 to 120 days (October 2019 to January 2020) in south Indian tertiary care hospital (Rajiv Gandhi Institute of Medical Sciences) Kadapa. A total of 60 patients were included in our study based on inclusion criteria.

\section{Distribution of Patients Based on Age}

We categorized the patients with respect to their age groups. Out of 60 patients majority i.e., $22(36.67 \%)$ between the age group of $41-50$ years, $18(30 \%)$ in between $31-40$ years, $9(15 \%)$ in between 21-30 years, $6(10 \%)$ in between $51-60$ years and very less $5(8.33 \%)$ in between age group of $61-70$ years, which was represented in below figure.

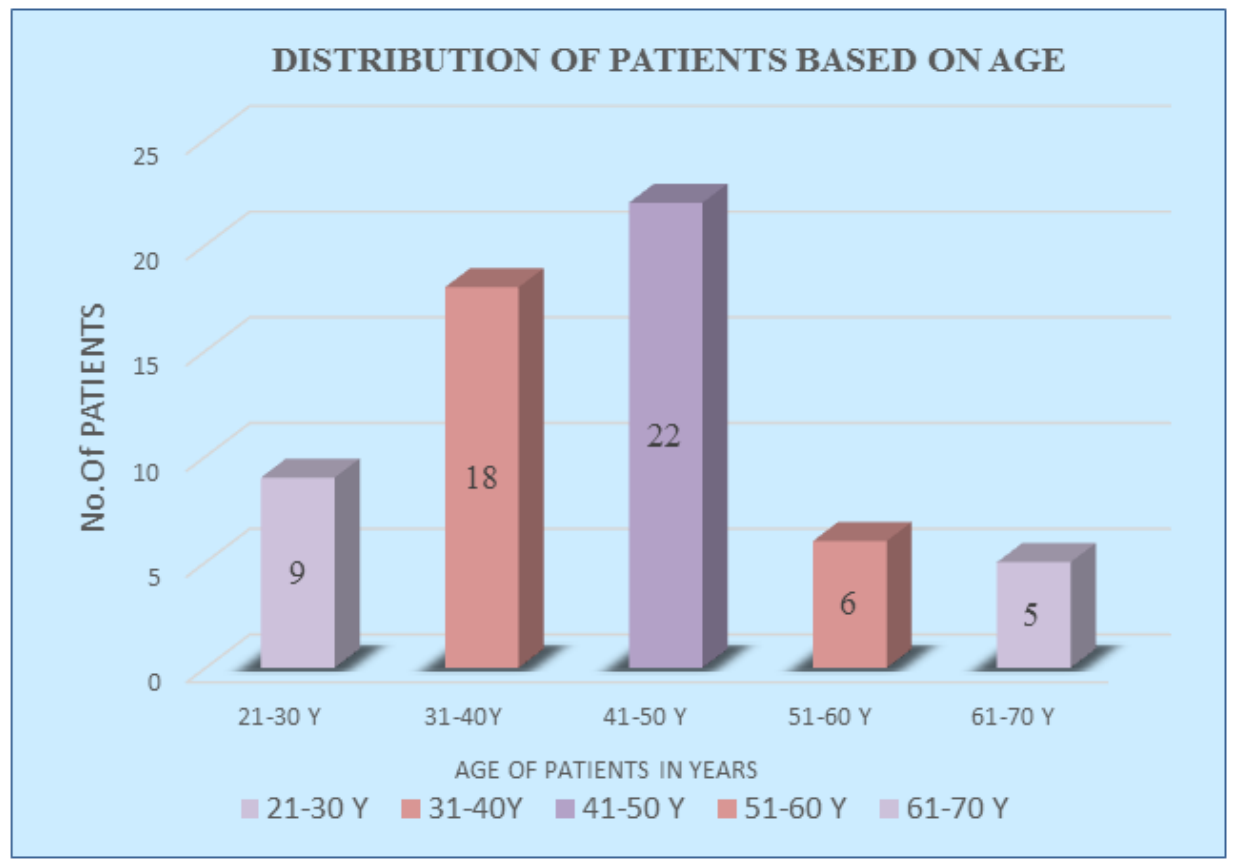

Fig-2: Distribution of patients based on age

\section{Distribution of Patients Based on Gender}

Out of 60 patients $45(75 \%)$ were males, $15(25 \%)$ patients were females and their ratio was 3:1 which was represented in below figure. 


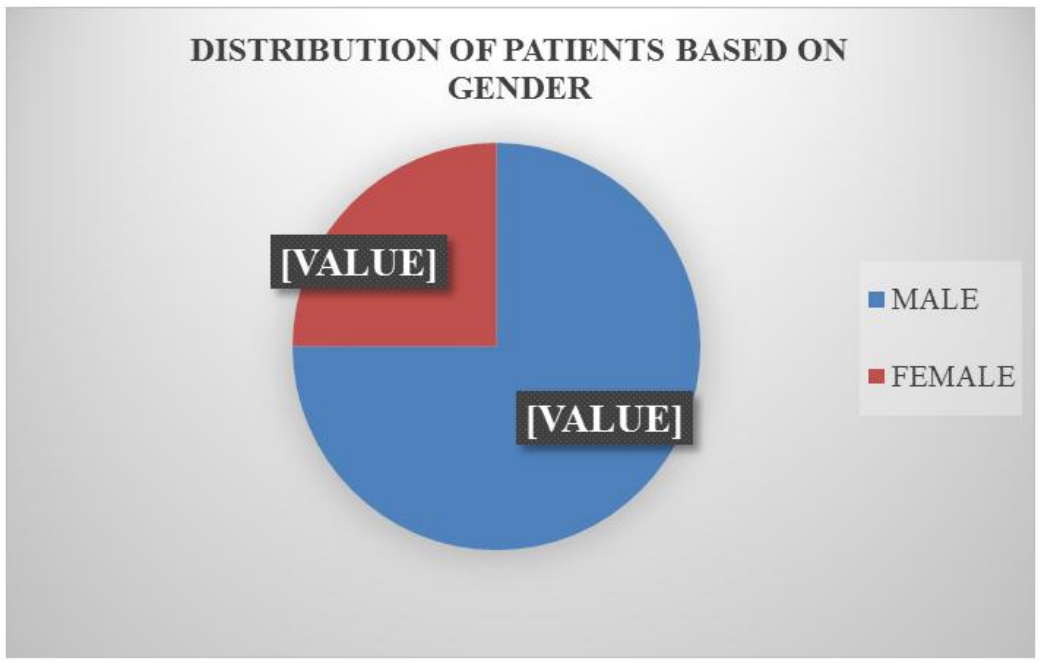

Fig-3: Distribution of patients based on gender

\section{Distribution of Patients Based on Social Habits}

Out of 60 patients, majority i.e., 34(56.67\%)

patients were with social habits (either smoking or alcohol or both) and $26(43.33 \%)$ were without social habits which are represented in below figure.

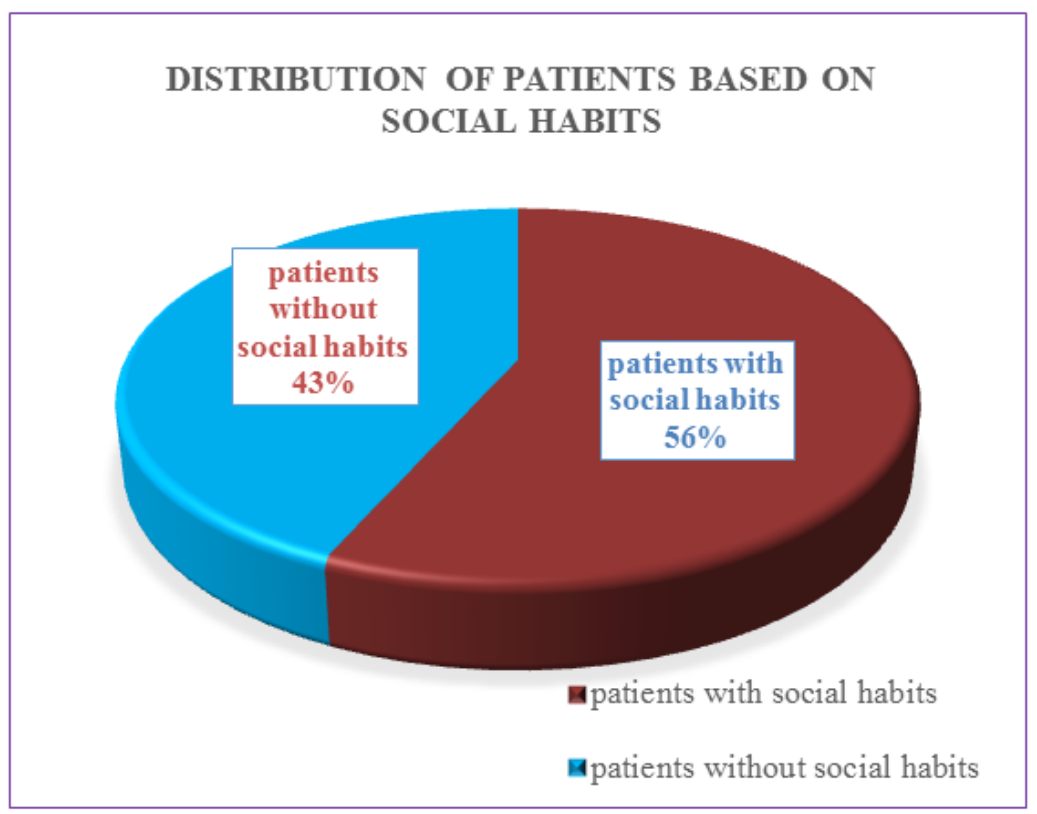

Fig-4: Distribution of patients based on social habits

Distribution of patients based on the admitting diagnosis

Out of 60 patients majority i.e., 27(45\%) patients were diagnosed with fractures, $9(15 \%)$ with osteomyelitis, $7(11.66 \%)$ with rheumatoid arthritis, $6(10 \%)$ with lower backache, $6 \quad(10 \%)$ with osteoarthritis and very less 5(8.33\%) with Spondylosis which was represented in below figure and table.

Table-1: Shows distribution of patients based on admitting diagnosis

\begin{tabular}{|l|l|l|}
\hline Admitting Diagnosis & Frequency & Percentage \\
\hline Rheumatoid Arthritis & 7 & 11.66 \\
\hline Osteomyelitis & 9 & 15 \\
\hline Fractures & 27 & 45 \\
\hline Lower Backache & 6 & 10 \\
\hline Spondylosis & 5 & 8.33 \\
\hline Osteoarthritis & 6 & 10 \\
\hline
\end{tabular}




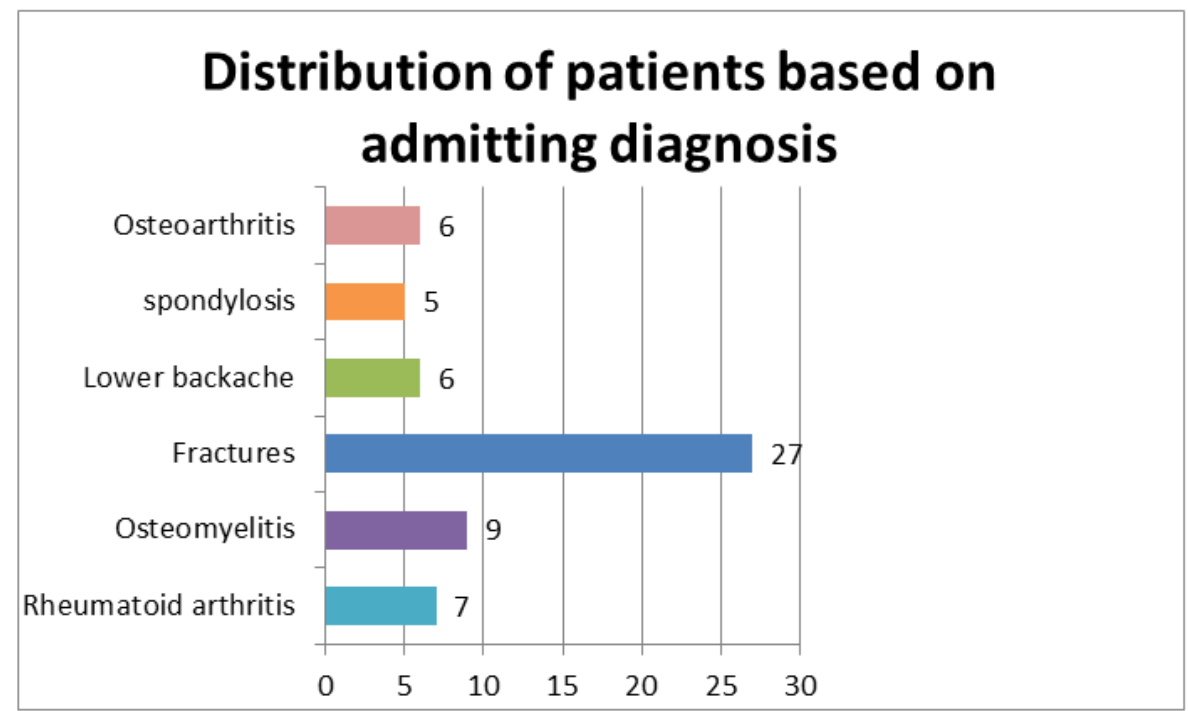

Fig-5: Distribution of patients based on admitting diagnosis

Distribution of patients based on the Comorbidities Out of 60 patients 23 patients were with comorbidities i.e., $8(13.33 \%$ Patients have hypertension, 6(10\%) patients have diabetes, 5(13.33) patients have asthma, $4(6.67 \%)$ patients have COPD.

Co prescribed drugs with diclofenac for patient during hospitalisation at a tertiary care hospital

Table-2: Shows Co prescribed drugs with diclofenac

\begin{tabular}{|l|l|}
\hline Category of drugs & Utilization of drugs \\
\hline Proton Pump Inhibitors (Pantoprazole) & $36(60 \%)$ \\
\hline $\mathrm{H}_{2}$ blockers (ranitidine) & $24(40 \%)$ \\
\hline Antimicrobials & $9(15 \%)$ \\
\hline Calcium supplements & $53(88.33 \%)$ \\
\hline Multi vitamins & $47(78.33 \%)$ \\
\hline Corticosteroids & $24(40 \%)$ \\
\hline Serratiopeptidase & $29(48.33 \%)$ \\
\hline Methotrexate & $9(15 \%)$ \\
\hline
\end{tabular}

Distribution of patients based on the occurrence of ADRs

Table-3: Shows distribution of patients based on the occurrence of ADRs

\begin{tabular}{|l|l|l|}
\hline S. No & ADRs & Total no. of patients \\
\hline 1. & Nausea & 2 \\
\hline 2. & Vomiting & 1 \\
\hline 3. & Gastritis & 3 \\
\hline 4. & Abdominal discomfort & 2 \\
\hline 5. & Diarrhea & 1 \\
\hline 6. & Headache & 1 \\
\hline
\end{tabular}

Causality assessment of ADRs based on WHO causality assessment scale

Table-4: Shows Causality assessment of ADRs based on WHO scale

\begin{tabular}{|l|l|l|l|l|}
\hline S. No & ADRs & $\begin{array}{l}\text { No. of ADRs } \\
\text { (Possible) }\end{array}$ & $\begin{array}{l}\text { No. of ADRs } \\
\text { (Probable) }\end{array}$ & $\begin{array}{l}\text { No. of ADRs } \\
\text { (Certain) }\end{array}$ \\
\hline 1. & Nausea & 1 & 1 & \\
\hline 2. & Vomiting & 1 & & \\
\hline 3. & Gastritis & 2 & 1 & \\
\hline 4. & Abdominal discomfort & 1 & 1 & \\
\hline 5. & Diarrhea & 1 & & \\
\hline 6. & Headache & 1 & & \\
\hline & & $7(11.67 \%)$ & $3(5 \%)$ & $10(16.67 \%)$ \\
\hline
\end{tabular}


Severity assessment of ADRs based on Hartwig Siegel scale

Out of 10 ADRs majority of the ADRs i.e., $10(16.67 \%)$ were mild in nature, and moderate and severe ADRs were not identified.

\section{Statistical Analysis}

The data from the data collection sheet were evaluated for various parameters. Descriptive statistics such as frequencies, percentages and means were used in the analysis of the data.

\section{DISCUSSION}

Our study endeavours to analyse the prescribing pattern of diclofenac in the Orthopaedics department and supplies baseline information about the prescribing practices with an aim to enhance rational prescribing. The evaluation of drugs prescribing patterns is considered essential to improve clinical and economical outcomes [6]. The present study regarding the pattern of NSAID (Diclofenac) prescribing in IP patient orthopaedic practice revealed that the nonarthritic indications were more common than the arthritic conditions. Fractures $(45 \%)$ and Osteomyelitis $(15 \%)$, were the most common indications for NSAID prescribing, which was consistent with the observations in other similar studies. Other classes of drugs used concomitantly were PPIs, H2receptor antagonists, calcium supplements, multivitamins, antimicrobials, Corticosteroids, Serratiopeptidase and Methotrexate which were used as separate combinations [7, 8]. The PPIs used were Pantoprazole, concomitant administration of PPIs with NSAIDs can be considered rational and justified as these classes of drugs are most effective in countering NSAID related ulcerogenicity. Calcium preparations and multivitamins were used as nutritional supplements. Methotrexate was used as DMARD in five subjects with RA to prevent the cartilage erosion.

The present study has reported the incidence of ADRs to diclofenac in the orthopaedic in-patient setting in the Indian scenario. According to our study, the incidence of ADRs was found to be $5.5 \%$. A recent study on the adverse drug reactions of non- steroidal anti-inflammatory drugs in orthopaedic patients in a tertiary care teaching hospital, Delhi, showed that the prevalence rate of the ADRs was $26 \%$. Our study showed a lower incidence of ADRs as compared to the findings of the above studies.

Of the patients who experienced ADRs during the study period, $7(63.63 \%)$ were males and 3 $(36.37 \%)$ were females. Reports from various studies showed a female preponderance, while our study did not show much difference with respect to gender, as our study had only limited number of patients.
Epidemiological studies showed that gastrointestinal tract (GIT) complications were the most common ADRs with ketoprofen, piroxicam and diclofenac [9]. One study reported that $20 \%$ of the patients experienced side effects due to Tab. Diclofenac [10]. As our study was carried out in the orthopaedic department, the ADRs which were caused by NSAIDs particularly diclofenac was studied, where 10 cases reported ADRs due to Tab. Diclofenac out of 60 prescriptions (16.67\%). In our setup, only low risk drugs were prescribed, which were related to gastro protection. Gastro protective agents were also given in $100 \%$ of the total patients, which minimized the GIT complications in our study, as has been described in evidence based medicine.

As per WHO causality assessment scale, $11.67 \%$ ADRs were assessed as "possible" ADRs and $5 \%$ as "probable" ADRs. The severity assessment scale revealed that $100 \%$ were mild and that moderate and severe ADRs were not identified as diclofenac was prophylactically prescribed with PPIs or $\mathrm{H} 2$ blockers [11].

A study which was conducted by Arulmani et al., on ADR monitoring in a secondary care hospital in South India, showed that $53.7 \%$ were mild ADRs, whereas our study showed more number of mild cases. No severe ADRs were found in our study [12].

In a recent study review, Peer Mohamed et al reported ADR frequencies between 10 and $20 \%$ in inpatients [13].

A number of studies have described NASIDs as the leading causes of ADRs, while others have shown that they ranked 4th or 5th in causing ADRs. Two studies by Chan denotes that NSAIDs were responsible for $28 \%$ of the drug related admissions in Hong Kong and that NSAIDs related GIT bleeds were about $18 \%$ in an another study. In a Scottish study, 17 patients reported ADRs due to NSAIDs [14]. Our study showed that GIT side effects were common and that they were mild in nature. When compared with the above studies, the incidence rate of the adverse drug effects in our orthopaedic in-patients was $16.67 \%$, which was lower than that which was found in the above mentioned studies.

As our study involved spontaneous reporting for adverse effects, we could detect 10 adverse effects. The treating doctors were considering risk factors like peptic ulcer in the patients. They prescribed drugs to prevent adverse effects whenever they anticipated adverse effects. So, the adoption of rational drug therapy by the treating doctors in a tertiary care teaching hospital where the study was done, might have contributed to the lower incidence of the adverse effects. 
The prevention of adverse reactions by identifying persons who are at a high risk is important to improve patient care. In our study, we found only a $16.67 \%$ incidence of adverse reactions which were caused due to rational drug uses. Despite the study being limited to one department, it has provided baseline data for further larger studies and it has ascertained the importance of prospective ADR monitoring in pharmacovigilance studies.

\section{CONCLUSION}

The present study has reported the prescribing pattern of diclofenac along with severity of ADRs among the orthopaedic in-patients. Overall, the incidence rate of ADRs in the orthopaedic ward was less and the ADRs reported were generally mild in severity and involved gastrointestinal tract. It showed that rational drug therapy had brought down the adverse effects to minimal and that it had attributed to the better prescription practice which was followed in our tertiary care hospital. Knowledge of potential adverse effects and standard prescription guide lines will play pivotal role in rational prescription of NSAIDs (diclofenac). Regular prescription audits with feedback to prescribers can alter prescribing behaviour towards good prescribing practices.

Our study concludes that there should be a continuous monitoring of the prescribing patterns of diclofenac by the clinical pharmacist to resolve the issues of inappropriateness and to enhance the rational prescribing. The clinical pharmacist plays a vital role in monitoring the adverse drug reactions of diclofenac which helps to prevent the occurrence of further complications.

\section{ACKNOWLEDGEMENT}

It is a great pleasance to convey our gratification and sincere thanks to patients and health care professionals, Department of orthopaedics, Govt. General Hospital, Kadapa for their guidance, supervision and support during the study. We also take this opportunity to acknowledge the principal, faculty and management of P. Rami Reddy Memorial College of Pharmacy, Kadapa for providing necessary facilities and timely help to complete our work.

Conflicts of Interest: There are no conflicts of interest.

\section{ABBREVATIONS}

ADR: Adverse Drug Reaction; CDSCO: Central Drug Standard Control Organization; COX: Cyclooxygenase; CVS: Cardio Vascular System; DMARD: Disease Modifying Anti Rheumatic Drugs; GIT: Gastro Intestinal Tract; IP: In-Patients; LBP: Lower Back Pain; NSAIDs: Non-Steroidal Anti Inflammatory Drugs; OP: Out-Patients; OA: Osteoarthritis; PPI: Proton Pump Inhibitors; RA: Rheumatoid Arthritis; RUM: Rational Use of Medicines; WHO: World Health Organization.

\section{SUMMARY}

- Prescribing pattern studies are conducted to monitor, evaluate and if necessary, suggest modifications in the prescribing behaviour of medical practitioners to make medical care rational and cost effective.

- The use of diclofenac as an over-the-counter (OTC) product for short-term use at a low dose remains appropriate.

- Use of Diclofenac sodium as pre emptive analgesic agent enhances its effect and very few patients (i.e., 10 patients) were suffered from adverse drug reactions in orthopedic patients.

- The choice of drugs, the duration and the route chosen by Orthopaedician were appropriate in all the cases. The appropriateness was determined by the authors after consulting different sources in the drug information center and the college library.

\section{REFERENCES}

1. Jyothi R, Pallavi D, Pundarikaksha $H$, Sridharmurthy JN, Girish K. A study of prescribing pattern of Nonsteroidal antiinflammatory drugs in orthopaedic OPD at a tertiary care hospital. National Journal of Basic Medical Sciences; 2015, 4(1):71-74

2. Manohar VS, Vinay M, Jayasree T, Kishan PV, Ubedulla S, Dixit R. Prescribing pattern of gastroprotective agents with non-steroidal antiinflammatory drugs. Journal of pharmacology \& pharmacotherapeutics. 2013 Jan;4(1):59-60.

3. Sharma T, Dutta S, Dhasmana DC. Prescribing pattern of NSAIDs in orthopaedic OPD of a tertiary care teaching hospital in Uttaranchal. JK Sci. 2006;8(3):160-2.

4. Choudhury DK, Bezbaruah BK. Prescribing pattern of analgesics in orthopedic in-patient department at tertiary care hospital in Guwahati, Assam, Northeast India. Indian journal of pharmacology. $2016 \mathrm{Jul}$;48(4):377-381.

5. Venkatachalam S, Bhat R. ADR Monitoring of NSAIDs among the in-patients of the orthopaedic ward in a Tertiary care centre: A prospective observational study. Journal of clinical and diagnostic research. 2012;1:42-6.

6. Alshakka MA, Badullah WF, Alolayan SO, Mahmoud MA. Prescribing patterns of nonsteroidal anti-inflammatory drugs (NSAIDs) at outpatient departments of four hospitals. Biomedical Research. 2018 Nov 5;29(19):15-25.

7. Shehnaz SI, Arifulla M, Jayadevan Sreedharan KG .Prescribing patterns in the Orthopedics outpatient department in GMC Hospital, Ajman, United Arab Emirate. Proceedings of the 6th Annual Scientific Meeting of Gulf Medical University. 2014; 8(2): 120-131 
8. R. Latha, K.Sreenivasu, M.Ananda Babu Naik, Jaya chandra Reddy. A study of prescribing pattern of non-steroidalanti-inflammatory drugs in orthopaedic outpatient department at a tertiary care hospital. J of evolution of Med and Dent Sci. 2015; 4(4): 559-564

9. Grosser T, Smyth E, Garret A, Fitz Gerald. Chapter 34, Anti-inflammatory, Antipyretic and Analgesic agents, Pharmacotherapy of Gout. In: Laurence. L. Bruton. PhD, Bruce A. Chabuer, editors. Goodman and Gilman's The Pharmacological Basis of Therapeutics, 12th edition. New York, McGraw-Hill; 2011; 966-993.

10. Larmour I, Dolphin RG, Baxter H, Morrison S, Hooke DH, Mc Grath BP. A prospective study of hospital admissions due to drug reactions. Australian J Hosp Pharm. 1991; 21(2): 90-95.

11. Batta A, Madan N, Kalra BS, Arora S. Prescription Audit, Drug Utilization Pattern and
Adverse Drug Reaction Monitoring in Outpatients of Orthopedics Department of Tertiary Care Teaching Hospital: A Pilot Study. MAMC Journal of Medical Sciences. 2019 May 1;5(2):77.

12. Arulmani R, Rajendran SD, Suresh B. Adverse drug reaction monitoring in a secondary care hospital in South India. Br J Clin Pharm. 2008; 65(2): 210-216

13. Gor AP, Desai SV. Adverse drug reactions in the inpatients of the medicine department of a tertiary care teaching hospital and the influence of pharmacovigilance in the reporting of ADRs. Indian J Pharmacol. 2008; 4(10):37-40.

14. Cunnngham G, Dodd TRP, Grant DT, Murdo MET, Richards RME. Drug related problems in elderly patients who were admitted to Tayside hospitals, methods for prevention and subsequent reassessment. Age and Ageing. 1997; 2(6):375382. 